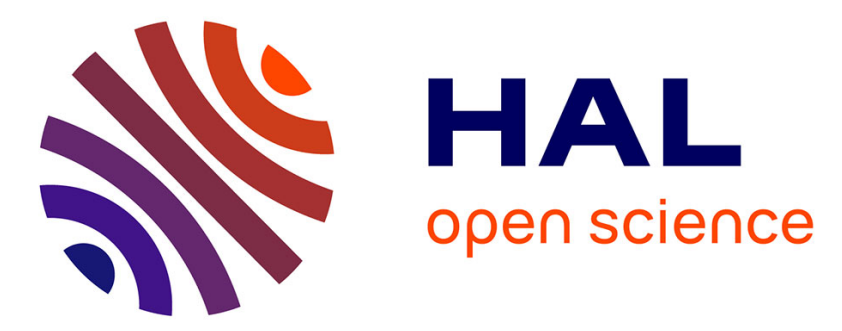

\title{
Long-term prognosis for transplant-free survivors of paracetamol-induced acute liver failure
}

Peter Jepsen, Lars E. Schmidt, Fin Stolze Larsen, Hendrik Vilstrup

\section{To cite this version:}

Peter Jepsen, Lars E. Schmidt, Fin Stolze Larsen, Hendrik Vilstrup. Long-term prognosis for transplant-free survivors of paracetamol-induced acute liver failure. Alimentary Pharmacology and Therapeutics, 2010, 32 (7), pp.894. 10.1111/j.1365-2036.2010.04419.x . hal-00561324

\section{HAL Id: hal-00561324 https://hal.science/hal-00561324}

Submitted on 1 Feb 2011

HAL is a multi-disciplinary open access archive for the deposit and dissemination of scientific research documents, whether they are published or not. The documents may come from teaching and research institutions in France or abroad, or from public or private research centers.
L'archive ouverte pluridisciplinaire HAL, est destinée au dépôt et à la diffusion de documents scientifiques de niveau recherche, publiés ou non, émanant des établissements d'enseignement et de recherche français ou étrangers, des laboratoires publics ou privés. 


\begin{tabular}{l}
\hline Alimentary Pharmacology \\
\hline \& Therapeutics \\
\hline
\end{tabular}

\section{Long-term prognosis for transplant-free survivors of paracetamol-induced acute liver failure}

\begin{tabular}{|c|c|}
\hline Journal: & Alimentary Pharmacology \& Therapeutics \\
\hline Manuscript ID: & APT-0187-2010.R1 \\
\hline Wiley - Manuscript type: & Original Scientific Paper \\
\hline $\begin{array}{r}\text { Date Submitted by the } \\
\text { Author: }\end{array}$ & 27-Jun-2010 \\
\hline Complete List of Authors: & $\begin{array}{l}\text { Jepsen, Peter; Aarhus University Hospital, Department of Medicine } \\
\text { V (Hepatology and Gastroenterology); Aarhus University Hospital, } \\
\text { Department of Clinical Epidemiology } \\
\text { Schmidt, Lars; Rigshospitalet,, Department of Hepatology A } \\
\text { Larsen, Fin; Rigshospitalet, Department of Hepatology A } \\
\text { Vilstrup, Hendrik; Aarhus University Hospital, Department of } \\
\text { Medicine V (Hepatology and Gastroenterology) }\end{array}$ \\
\hline Keywords: & $\begin{array}{l}\text { Epidemiology < Topics, Drug-induced liver disease < Hepatology, } \\
\text { Liver < Organ-based, X keyword = no topic }\end{array}$ \\
\hline
\end{tabular}

\section{ScholarONE \\ Manuscript Central}




\title{
Long-term prognosis for transplant-free survivors of paracetamol-
} induced acute liver failure

\author{
Peter Jepsen $^{1,2}$, MD \\ Lars E. Schmidt ${ }^{3}$, MD \\ Fin Stolze Larsen ${ }^{3}, \mathrm{MD}$ \\ Hendrik Vilstrup ${ }^{1}$, professor
}

1. Department of Medicine V (Hepatology and Gastroenterology), Aarhus University Hospital, Aarhus, Denmark.

2. Department of Clinical Epidemiology, Aarhus University Hospital, Aarhus, Denmark.

3. Department of Hepatology, Rigshospitalet, University Hospital of Copenhagen, Copenhagen, Denmark.

Running title: Prognosis for survivors of acute liver failure.

Corresponding author: Peter Jepsen, Department of Medicine V (Hepatology and Gastroenterology), Aarhus University Hospital, Nørrebrogade, 8000 Aarhus C, Denmark. Telephone: +45 8949 3892. Fax: +45 8942 4801; E-mail: pj@ dce.au.dk.

Keywords: Fulminant liver failure; acetaminophen; hepatic encephalopathy; mortality; survival; epidemiology.

Abbreviations: $\mathrm{CI}=$ Confidence interval; $\mathrm{ICD}=$ International Classification of Diseases. 


\section{Summary \\ Background: The prognosis for transplant-free survivors of paracetamol-induced acute liver failure is unknown.}

Aim: To examine whether paracetamol-induced acute liver failure increases long-term mortality.

Methods: We followed all transplant-free survivors of paracetamol-induced acute liver injury hospitalized in a Danish national referral center during 1984-2004. We compared age-specific mortality rates from one year post-discharge through 2008 between those in whom the liver injury led to an acute liver failure and those in whom it did not.

Results: We included 641 patients. On average, age-specific mortality rates were slightly higher for the 101 patients whose paracetamol-induced liver injury had caused an acute liver failure (adjusted mortality rate ratio $=1.70,95 \% \mathrm{CI} 1.02-2.85)$, but the association was age-dependent, and no survivors of acute liver failure died from liver disease, whereas suicides were frequent in both groups. These observations speak against long-term effects of acute liver failure. More likely, the elevated mortality rate ratio resulted from incomplete adjustment for the greater prevalence of substance abuse among survivors of acute liver failure.

Conclusions: Paracetamol-induced acute liver failure did not affect long-term mortality. Clinical follow-up may be justified by the cause of the liver failure, but not by the liver failure itself. 


\section{Materials and methods}

\section{Study population}

According to national guidelines, Danish citizens hospitalized with severe paracetamol-induced liver injury (i.e. with the International Normalized Ratio [INR] above 1.7) must be considered for transfer to The Liver Unit at Rigshospitalet, Copenhagen, Denmark's only liver transplantation 
center. The decision to transfer patients is made by specialists at The Liver Unit on an individual basis. We identified all patients who were hospitalized for paracetamol-induced liver injury in The Liver Unit during 1984-2004, had an INR above 1.7 but survived without a liver transplant and were still alive one year after discharge. We had no data on other patients. Among the 656 identified patients we excluded 15 who at the time of the paracetamol-induced liver injury had chronic liver disease, identified through the Danish National Patient Registry which has recorded hospital contacts in Denmark since 1977 (8). The registry data include admission and discharge dates and discharge diagnoses coded by physicians using the $8^{\text {th }}$ revision of the International Classification of Diseases (ICD-8) through 1993 and the $10^{\text {th }}$ revision (ICD-10) thereafter. Chronic liver disease was defined by a history of a diagnosis code for alcoholic cirrhosis (ICD-8: 571.09; ICD-10: K70.3), biliary cirrhosis (ICD-8: 571.90-91; ICD-10: K74.3-5), chronic hepatitis (ICD-8: 571.93, 573.02; ICD-10: B18.x, K73.x), or unspecific cirrhosis (ICD-8: 571.92, 571.99; ICD-10: K74.0-2, K74.6). Simultaneous overdose of drugs other than paracetamol was not a criterion for inor exclusion.

\section{Patient data}

We used data from the patients' medical charts to ascertain the diagnosis of acute liver failure, defined by coagulopathy, jaundice, and hepatic encephalopathy grade 2-4 (9). We also obtained information on presence or absence of alcohol abuse; other substance abuse; schizophrenia or use of antipsychotic medication; affective disorder or use of antidepressants; borderline personality disorder; and eating disorder. We supplemented the chart data with discharge diagnoses of the same conditions in the National Patient Registry: alcohol abuse (ICD-8: 291.xx, 303.xx except 303.90; ICD-10: F10.2-9), other substance abuse (ICD-8: 294.30, 294.38, 304.xx; ICD-10: F11.x to F19.x), schizophrenia (ICD-8: 295.xx; ICD-10: F2x.x), affective disorder (ICD-8: 296.xx, 298.xx; ICD-10: F3x.X), borderline personality disorder (ICD-8: none; ICD-10: F60.3), and eating disorder (ICD-8: 


\section{Mortality data}

Dates of death were obtained from the Central Office of Civil Registration, where data are updated continuously with no loss to follow-up (10). Causes of death were ascertained from the Cause of Death Registry, which contains information on up to four events or medical conditions that led to death for each decedent. The data are supplied by the physician responsible for the patient's treatment at the time of death (11). We reviewed the registered data to define a single cause of death for each patient; the investigators were blinded to patients' acute liver failure status.

\section{Statistical analyses}

Follow-up began one year after the date of discharge following paracetamol-induced liver injury and ended at death or on 31 December 2008, whichever came first. The 1-year delay in follow-up was introduced to avoid bias due to early deaths related to the poisoning or its psychiatric cause (e.g. risk of completed suicide is particularly high up to one year after a paracetamol poisoning) (12). We used the Mann-Whitney test for the continuous variables, i.e. patient age and calendar time, and Pearson's chi-square test for the other variables to test the hypotheses that patients with or 
without acute liver failure had similar characteristics at inclusion. We used a p-value below 0.05 to denote statistically significant non-similarity.

We compared the mortality rate ratio (specifically, the hazard ratio) for patients with and without acute liver failure using Cox proportional hazards regression, and we adjusted for the effects of gender, calendar year, alcohol abuse, other substance abuse, schizophrenia or use of antipsychotics, affective disorder or use of antidepressants, borderline personality disorder, eating disorder, cancer, chronic obstructive lung disease, diabetes, arterial hypertension, and ischemic heart disease. Because we followed patients from a time point defined by recovery from acute paracetamolinduced liver injury, we anticipated that patient age was a stronger predictor of death than time since hospital discharge, and therefore a more relevant time scale for our analyses (13). Using natural splines, we plotted the age-specific adjusted mortality rate ratios for acute liver failure (14).

Comparison with population mortality

Through the Central Office of Civil Registration we identified a control group from the general population, the aim being to substantiate the excess mortality for patients hospitalized for paracetamol-induced liver injury. We included 100 gender-, age-, and calendar time-matched Danish citizens per patient without acute liver failure, and they were drawn at random using risk set sampling (15). Using Cox regression and natural splines, as described above, we plotted the agespecific mortality rate ratios for the general population sample vs. patients with paracetamolinduced liver injury without liver failure.

\section{Results}

We included 641 transplant-free survivors of paracetamol-induced liver injury, and $101(16 \%)$ of these had acute liver failure. Among the patients with acute liver failure, $16 \%$ had hepatic encephalopathy of grade $2 ; 51 \%$ of grade 3 ; and $33 \%$ of grade 4 . The median follow-up time was 
7.6 years, the maximum 23.2 years; 86 patients (13\%) died during the follow-up, including 23 patients in the acute liver failure group.

Patients with acute liver failure were more likely than patients without acute liver failure to be female, and they were older, and more likely to abuse alcohol and other substances (Table 1). Also the age-specific prevalence of substance abuse was higher among patients with a history of acute liver failure than among patients without such history. For example, at age 45 the prevalence of alcohol abuse was $58 \%$ in the acute liver failure group (14 of 24 patients under observation) compared with $29 \%$ in the group with milder liver injury (29 of 101 patients under observation) (Table 2).

On average, age-specific mortality rates were higher for patients with a history of acute liver failure (adjusted mortality rate ratio $=1.70,95 \%$ CI 1.02 to 2.85 ). However, the mortality rate ratio was strongly age-dependent, so that 25-, 45-, or 75-year-old people who had more than one year previously been hospitalized with paracetamol-induced acute liver failure had higher mortality than equal-age people who had more than one year previously been hospitalized with paracetamolinduced acute liver injury without liver failure, and vice versa among 35- or 60-year-old people (Figure 1). However, the earlier temporary failure of liver function was not the cause of the excess mortality: no patient with a history of acute liver failure was recorded to die from liver disease (data on causes of death were unavailable for three decedents). By contrast, six patients without acute liver failure died from acute or chronic liver disease. Suicides were very frequent in both groups (26 suicides $=37 \%$ of the 76 deaths with data on causes of death), as were accidents, cancer, and cardiovascular disease (Table 3).

Mortality before age 60 years was clearly higher for patients with a history of paracetamol-induced liver injury without acute liver failure than for the general population sample (Figure 1). Hence, the 
mortality in the general population was not a relevant estimate of the expected mortality among the $91 \%$ of our patients who were younger than 60 years old at discharge after paracetamol-induced liver injury.

\section{Discussion}

We followed transplant-free survivors of paracetamol-induced liver injury and found that those who had suffered an acute liver failure had marginally higher mortality, but for causes not attributable to their earlier liver failure. We also showed that survivors of paracetamol-induced liver injury younger than 60 years of age had a markedly higher mortality than the general population, largely due to suicides and accidents.

It is a long-standing and basic assumption that there is complete clinical recovery after a reversible loss of liver function. This assumption is rooted in the well-known regenerative capacity of the liver, but it has not previously been challenged by a long-term clinical follow-up study. Our data support the classical assumption. We found a marginal increase in mortality after acute liver failure, but it was age-dependent and not due to liver-related deaths. Rather, we expect the association to be an overestimate due to incomplete control of confounding by substance abuse, which was only crudely measured as absent or present.

The validity of the clinical diagnoses of acute liver failure is crucial because invalid diagnoses would have led us to underestimate the true effect of acute liver failure (16). However, we believe that the diagnoses were valid because they were made by specialists in a liver transplantation center and required clinically overt hepatic encephalopathy, i.e. grade 2-4.

The findings in our study accord with a recent follow-up study in which patients with and without hepatotoxic paracetamol overdose had parallel survival curves from one year after hospital 
discharge (17). In that study, however, at most only 15 deaths were observed more than one year after hospital discharge. Likewise, a study of post-discharge liver biopsies from patients with paracetamol-induced liver injury supplied histological evidence of reversibility (6), and complete regeneration has also been described after surgical liver injury in animals and humans $(4,5)$ and after severe toxic liver injury in rats (18).

Our comparison with mortality in the general population is consistent with our previous findings that, for several years after hospitalization for paracetamol poisoning, the risks of being diagnosed with a psychiatric disease and/or completing suicide are greatly elevated $(12,19)$. Our analyses also demonstrate that a general population sample is not the proper comparison group for evaluating long-term effects of acute liver injury: those pre-existing patient characteristics that lead to experiencing an acute liver injury, in casu from paracetamol overdose, heavily influence prognosis. Therefore we conducted the study within a cohort of patients with a history of paracetamol-induced liver injury and excluded those who died within a year post-discharge. These design features minimized confounding from patient characteristics such as suicidal intent as opposed to therapeutic misadventure, because we can expect similar characteristics- $82 \%$ with suicidal intent— in equalage Danish patients with or without acute liver failure after paracetamol poisoning (20).

In conclusion, paracetamol-induced acute liver failure did not increase long-term mortality, but many patients hospitalized for paracetamol-induced liver injury eventually completed suicide. Hence our findings reiterate that all patients hospitalized for paracetamol-induced liver injury should undergo psychiatric evaluation and follow-up, whereas follow-up by hepatologists is not indicated. 


\section{References}

1. Møller LR, Nielsen GL, Olsen ML, Thulstrup AM, Mortensen JT, Sørensen HT. Hospital discharges and 30-day case fatality for drug poisoning: a Danish population-based study from 1979 to 2002 with special emphasis on paracetamol. Eur J Clin Pharmacol 2004; 59: 911-5.

2. Larson AM, Polson J, Fontana RJ, et al. Acetaminophen-induced acute liver failure: results of a United States multicenter, prospective study. Hepatology 2005; 42: 1364-72.

3. Stravitz RT, Kramer DJ. Management of acute liver failure. Nat Rev Gastroenterol Hepatol 2009; 6: 542-53.

4. Michalopoulos GK, DeFrances MC. Liver regeneration. Science 1997; 276: 60-6.

5. Fausto N. Liver regeneration and repair: hepatocytes, progenitor cells, and stem cells. Hepatology 2004; 39: 1477-87.

6. Portmann B, Talbot IC, Day DW. Histopathological changes in the liver following a paracetamol overdose: correlation with clinical and biochemical parameters. J Pathol 1975; 117: 169-81.

7. Björnsson E, Davidsdottir L. The long-term follow-up after idiosyncratic drug-induced liver injury with jaundice. J Hepatol 2009; 50: 511-7.

8. Andersen TF, Madsen M, Jørgensen J, Mellemkjær L, Olsen JH. The Danish National Hospital Register. A valuable source of data for modern health sciences. Dan Med Bull 1999; 46: $263-8$.

9. Polson J, Lee WM. AASLD position paper: the management of acute liver failure. Hepatology 2005; 41: 1179-97.

10. Frank L. When an entire country is a cohort. Science 2000; 287: 2398-9.

11. Juel K, Helweg-Larsen K. The Danish registers of causes of death. Dan Med Bull 1999; 46: 354-7. 
12. Qin P, Jepsen P, Nørgård B, et al. Hospital admission for non-fatal poisoning with weak analgesics and risk for subsequent suicide: a population study. Psychol Med 2009: 1-7.

13. Dam-Larsen S, Franzmann M, Andersen IB, et al. Long term prognosis of fatty liver: risk of chronic liver disease and death. Gut 2004; 53: 750-5.

14. Therneau TM, Grambsch PM. Testing proportional hazards. In: Modeling survival data: extending the Cox model. 1st ed. New York: Springer; 2000. p. 127-48.

15. Langholz B, Thomas DC. Nested case-control and case-cohort methods of sampling from a cohort: a critical comparison. Am J Epidemiol 1990; 131: 169-76.

16. Hutcheon JA, Chiolero A, Hanley JA. Random measurement error and regression dilution bias. BMJ 2010; 340: c2289.

17. Myers RP, Shaheen AA, Li B, Dean S, Quan H. Impact of liver disease, alcohol abuse, and unintentional ingestions on the outcomes of acetaminophen overdose. Clin Gastroenterol Hepatol 2008; 6: 918-25.

18. Fausto N, Campbell JS, Riehle KJ. Liver regeneration. Hepatology 2006; 43: S45-53.

19. Jepsen P, Qin P, Nørgård B, et al. The association between admission for poisoning with paracetamol or other weak analgesics and subsequent admission for psychiatric disorder: a Danish nationwide case-control study. Aliment Pharmacol Ther 2005; 22: 645-51.

20. Schmidt LE. Age and paracetamol self-poisoning. Gut 2005; 54: 686-90. 


\section{Declaration of interests}

This study was funded in part by Rigshospitalet; University of Copenhagen; The Laerdal

Foundation for Acute Medicine; The Sawmill Owner Jeppe Juhl and Wife Ovita Juhl Foundation

(Savværksejer Jeppe Juhl og Hustru Ovita Juhls Fond); The Novo Nordisk Foundation; The AP-

Møller Foundation; The Danish Hospital Foundation for Medical Research - Region of

Copenhagen, The Faroe Islands and Greenland; and The Danish Medical Association Research

Fund. The sponsors were not involved in any other way. 


\section{Figure legend}

Figure 1. Mortality rates for patients with a history of paracetamol-induced acute liver failure (black) and for a random population sample (gray) relative to that for patients with a history of paracetamol-induced liver injury without liver failure (the dotted horizontal line). The thin lines show $95 \%$ confidence limits. Hence the black line shows that at age 45-50 years, primarily, mortality was higher for patients with a history of paracetamol-induced acute liver failure than for patients with a history of paracetamol-induced liver injury without acute liver failure. The gray line shows that mortality before age 60 years was much lower for the general population sample than for patients with a history of paracetamol-induced liver injury without acute liver failure. The horizontal boxplots illustrate the age distribution (median, quartiles, and extremes) at the time of paracetamol-induced liver injury with or without acute liver failure (ALF), and the superimposed black dots mark the ages at which deaths occurred. 
Table 1. Characteristics of patients at inclusion. For the continuous variables, age and year of inclusion, the numbers shown are the median and $25^{\text {th }}$ and $75^{\text {th }}$ percentiles.

\begin{tabular}{lccc}
\hline & Acute liver failure & No acute liver failure & p-value \\
\hline Age & $43(33-55)$ & $35(24-47)$ & $<0.001$ \\
Women & $73(72 \%)$ & $331(61 \%)$ & 0.05 \\
Year of inclusion & $1999(1996-2003)$ & $2001(1996-2003)$ & 0.50 \\
Substance abuse & & & \\
Alcohol abuse & $47(47 \%)$ & $145(27 \%)$ & $<0.001$ \\
Other substance abuse & $14(14 \%)$ & $34(6.3 \%)$ & 0.01 \\
Psychiatric disease & & & 0.21 \\
Schizophrenia or use of antipsychotics & $16(16 \%)$ & $59(11 \%)$ & 0.61 \\
Affective disorder or use of antidepressants & $42(42 \%)$ & $207(38 \%)$ & 0.83 \\
Borderline personality disorder & $2(2.0 \%)$ & $12(2.2 \%)$ & 0.46 \\
Eating disorder & 0 & $8(1.5 \%)$ & 0.75 \\
Somatic disease & & & \\
Cancer & $101(100 \%)$ & $540(100 \%)$ & 0.72 \\
Chronic obstructive lung disease & $1(1.0 \%)$ & $4(0.74 \%)$ & 0.68 \\
Diabetes & $1(1.0 \%)$ & $6(1.1 \%)$ & $(2.8 \%)$ \\
Arterial hypertension & $3(3.0 \%)$ & $15(2.2 \%)$ & 0.53 \\
Ischemic heart disease & $4(4.0 \%)$ & & \\
\hline Total & $2(2.0 \%)$ & & \\
\hline
\end{tabular}


Table 2. Characteristics of the 641 patients with paracetamol-induced liver injury, by current age. Columns show the number of patients in the cohort who are observed at a particular age and their characteristics. Patients are followed from 1 year after discharge after a paracetamol-induced liver injury, so a patient who was 33 years old at discharge and died at age 49 is under observation from age 34 to age 49.

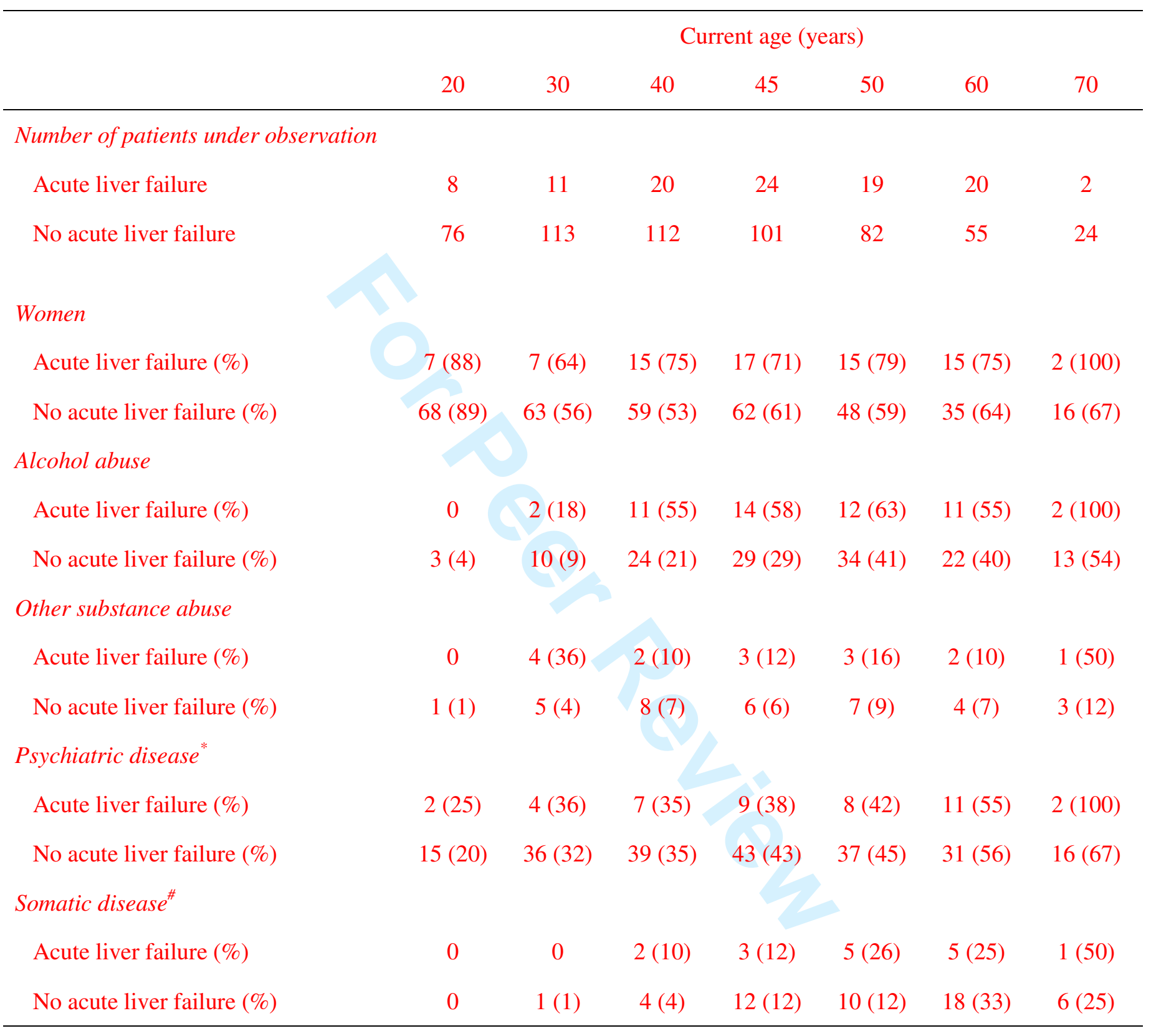

* Schizophrenia including use of antipsychotics, affective disorder including use of antidepressants, borderline personality disorder, or eating disorder.

\# Cancer, chronic obstructive lung disease, diabetes, arterial hypertension, or ischemic heart disease. 
Table 3. Causes of death. A total of 86 patients died during follow-up, but this table excludes the three patients with acute liver failure and seven patients without acute liver failure with no available data on cause of death.

\begin{tabular}{lcc}
\hline Cause of death & Acute liver failure & No acute liver failure \\
\hline Acute liver disease & 0 & $1(2 \%)$ \\
Chronic liver disease & 0 & $5(9 \%)$ \\
Alcoholism & 0 & $5(9 \%)$ \\
Cancer & $4(20 \%)$ & $6(11 \%)$ \\
Cardiovascular disease & $3(15 \%)$ & $7(13 \%)$ \\
Diabetes & $1(5 \%)$ & 0 \\
Pneumonia & 0 & $2(4 \%)$ \\
Respiratory disease & $2(10 \%)$ & 0 \\
Gastrointestinal bleeding & 0 & $1(2 \%)$ \\
Dementia & 0 & $1(2 \%)$ \\
Epilepsy & 0 & $1(2 \%)$ \\
Accident & $2(10 \%)$ & $7(13 \%)$ \\
Suicide & $8(40 \%)$ & $20(36 \%)$ \\
\hline Total & $20(100 \%)$ & $56(100 \%)$ \\
\hline
\end{tabular}




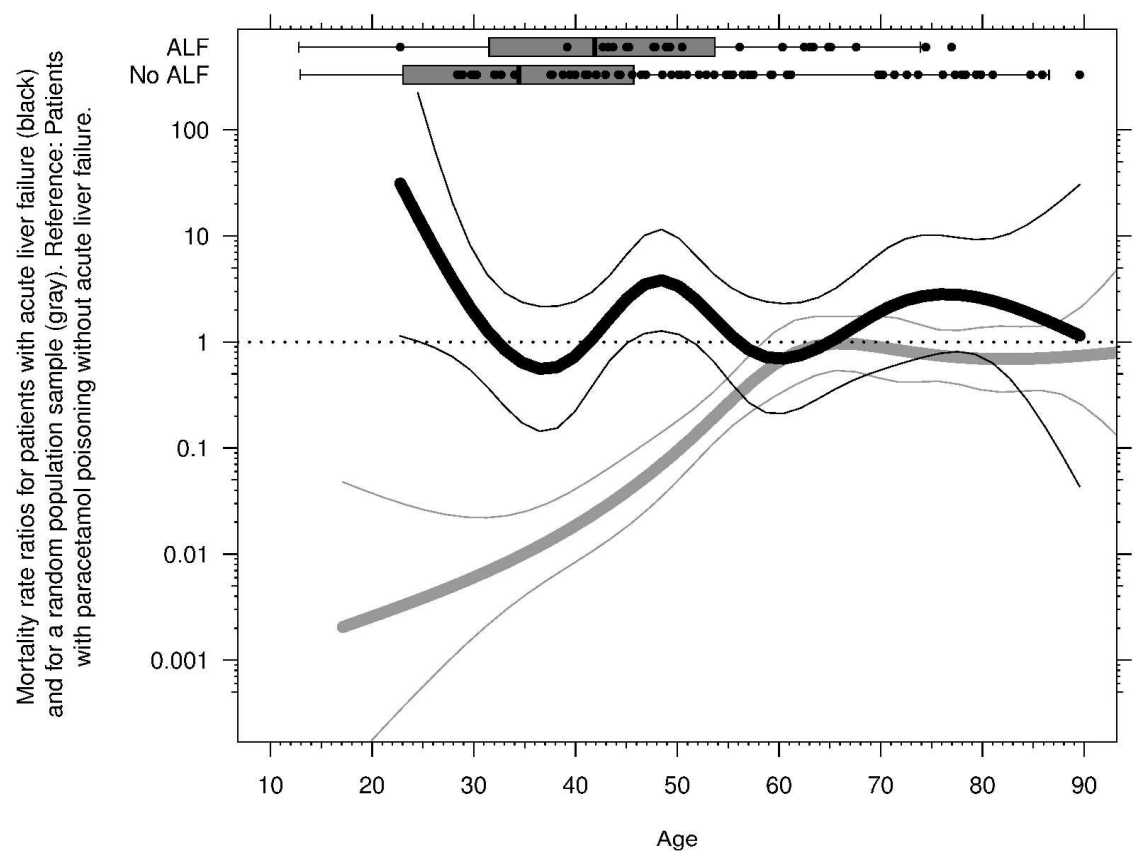

Figure 1. Mortality rates for patients with a history of paracetamol-induced acute liver failure (black) and for a random population sample (gray) relative to that for patients with a history of paracetamol-induced liver injury without liver failure (the dotted horizontal line). The thin lines show $95 \%$ confidence limits. Hence the black line shows that at age 45-50 years, primarily, mortality was higher for patients with a history of paracetamol-induced acute liver failure than for patients with a history of paracetamol-induced liver injury without acute liver failure. The gray line shows that mortality before age 60 years was much lower for the general population sample than for patients with a history of paracetamol-induced liver injury without acute liver failure. The horizontal boxplots illustrate the age distribution (median, quartiles, and extremes) at the time of paracetamol-induced liver injury with or without acute liver failure (ALF), and the superimposed black dots mark the ages at which deaths occurred. $215 \times 279 \mathrm{~mm}(600 \times 600 \mathrm{DPI})$ 
1

2

3

4

5

6

7

8

9

10

11

12

13

14

15

16

17

18

19

20

21

22

23

24

25

26

27

28

29

30

31

32

33

34

35

36

37

38

39

40

41

42

43

44

45

46

47

48

49

50

51

52

53

54

55

56

57

58

59

60 